\title{
Determination of the Elastic Behavior of Silicon Nanowires within a Scanning Electron Microscope
}

\author{
Nicole Wollschläger, ${ }^{1}$ Zuhal Tasdemir, ${ }^{2}$ Ines Häusler, ${ }^{1}$ Yusuf Leblebici, ${ }^{3}$ \\ Werner Österle, ${ }^{1}$ and B. Erdem Alaca, ${ }^{2,4}$ \\ ${ }^{1}$ Federal Institute of Materials Research and Testing (BAM), Unter den Eichen 87, 12205 Berlin, Germany \\ ${ }^{2}$ Department of Mechanical Engineering, Koç University, Rumelifeneri Yolu, 34450 Istanbul, Turkey \\ ${ }^{3}$ EPFL, Microelectronic Systems Laboratory, Building ELD, Station 11, 1015 Lausanne, Switzerland \\ ${ }^{4}$ Surface Science and Technology Center, Koç University, Rumelifeneri Yolu, 34450 Istanbul, Turkey
}

Correspondence should be addressed to Werner Österle; werner.oesterle@bam.de

Received 24 March 2016; Accepted 27 June 2016

Academic Editor: Philippe Caroff

Copyright (C) 2016 Nicole Wollschläger et al. This is an open access article distributed under the Creative Commons Attribution License, which permits unrestricted use, distribution, and reproduction in any medium, provided the original work is properly cited.

\begin{abstract}
Three-point bending tests were performed on double-anchored, $\langle 110\rangle$ silicon nanowire samples in the vacuum chamber of a scanning electron microscope (SEM) via a micromanipulator equipped with a piezoresistive force sensor. Nanowires with widths of $35 \mathrm{~nm}$ and $74 \mathrm{~nm}$ and a height of $168 \mathrm{~nm}$ were fabricated. The nanowires were obtained monolithically along with their $10 \mu \mathrm{m}$ tall supports through a top-down fabrication approach involving a series of etching processes. The exact dimension of wire cross sections was determined by transmission electron microscopy (TEM). Conducting the experiments in an SEM chamber further raised the opportunity of the direct observation of any deviation from ideal loading conditions such as twisting, which could then be taken into consideration in simulations. Measured force-displacement behavior was observed to exhibit close resemblance to simulation results obtained by finite element modeling, when the bulk value of $169 \mathrm{GPa}$ was taken as the modulus of elasticity for $\langle 110\rangle$ silicon. Hence, test results neither show any size effect nor show evidence of residual stresses for the considered nanoscale objects. The increased effect of the native oxide with reduced nanowire dimensions was captured as well. The results demonstrate the potential of the developed nanowire fabrication approach for the incorporation in functional micromechanical devices.
\end{abstract}

\section{Introduction}

Silicon-based microelectromechanical systems (MEMS) constitute one of the most promising fields, where the advantages of miniaturization are fully utilized to realize a variety of technical applications [1-3]. A new generation MEMS has recently evolved through the deterministic integration of microscale structures with silicon nanowires, a combination that leads to an enhancement in the overall functionality [4], specifically regarding the measurement resolution of small displacements through piezoresistive transduction [5]. The main challenge of this type of microscale or nanoscale integration is to meet the needs of monolithic fabrication, while nanowires remain intact during etching of extreme MEMS topographies. The monolithic fabrication is a robust and well established technique often used by industrial producers. Methods such as chemical etching [6] or growth through vapor-liquid-solid techniques [7] are either materialintensive with the problem of hazardous chemical waste disposal or cost-intensive and not stable for large area or batch fabrication. Hence, there is a consensus on the use of a top-down approach in applications, which have demanding registration and topography requirements.

Mechanical properties of electromechanical building blocks depend strongly on the specific fabrication techniques. Hence, a new fabrication technology would necessitate the exploration of the mechanical characteristics of the resulting structures. Answering this need becomes even more critical, if the structure of interest is a nanowire with very small dimensions making alignment and force measurement aspects of testing very challenging. In spite of the fact that a lot of investigations have been performed on silicon, mechanical 


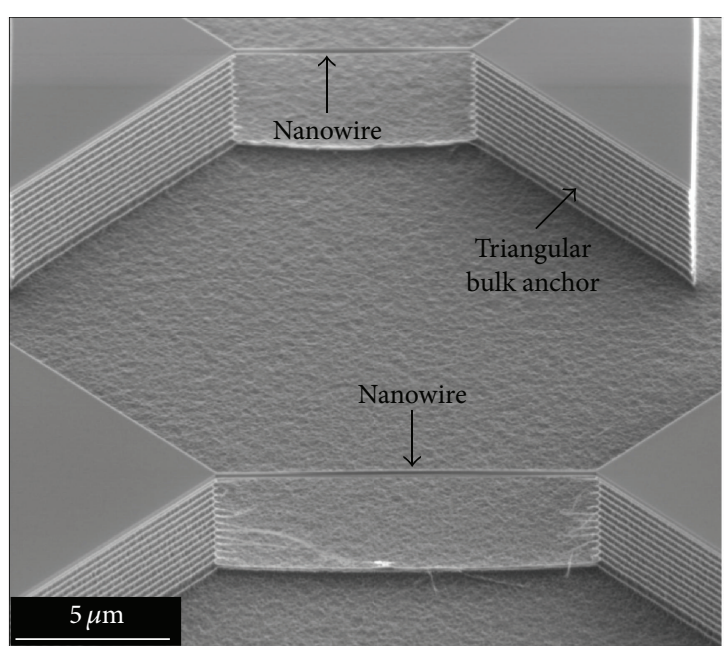

(a)

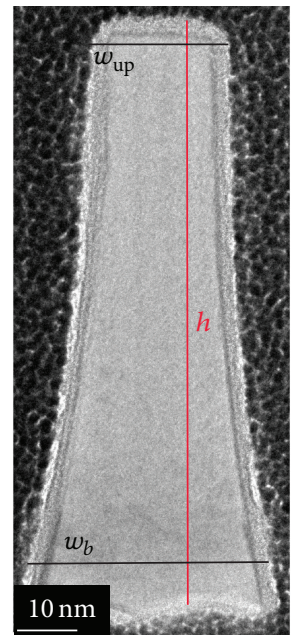

(b)

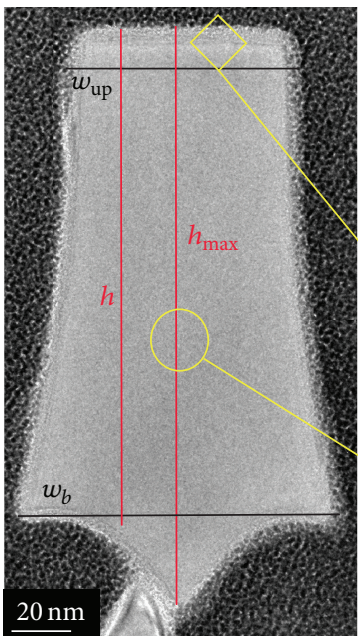

(c)

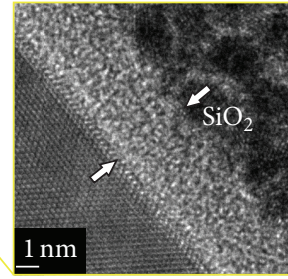

(c1)

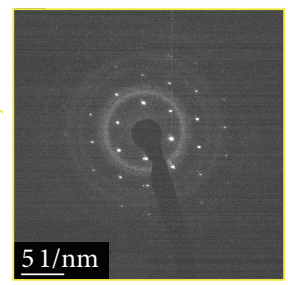

(c2)

FIGURE 1: Sample overview: (a) SEM image with double-anchored nanowires, where the ends are rigidly connected to triangular bulk anchors; (b) TEM cross-sectional image of the smallest silicon nanowire with a trapezoidal shape $\left(w_{\text {up }}=35 \mathrm{~nm}, w_{b}=66 \mathrm{~nm}\right.$, and $\left.h=168 \mathrm{~nm}\right)$; (c) TEM cross-sectional image of the largest silicon nanowire with a pentagonal shape ( $w_{\text {up }}=74 \mathrm{~nm}, w_{b}=112 \mathrm{~nm}, h=168 \mathrm{~nm}$, and $\left.h_{\max }=198 \mathrm{~nm}\right) ;(\mathrm{cl})$ high-resolution TEM image shows the native oxide layer; (c2) the diffraction pattern confirms the crystallinity and orientation of the wire.

measurements on very small structures are still rare. Most previous studies on silicon nanowires had to cope with the difficulty of positioning the nanoobjects by a combination of optical microscopy and AFM imaging [8-10]. Recently, the application of MEMS in combination with AFM and SEM was utilized for performing nanoscale tensile testing $[11,12]$ and diametrical compression testing [13]. In all these studies silicon nanowires were mechanically tested and similar elastic behavior as for bulk material was found for all objects with diameters bigger than $25 \mathrm{~nm}$. In consequence we were using the material bulk properties to validate our testing method. In this work we applied a new method of in situ bending within SEM on nanoobjects, which was shown to have three major advantages: (i) Due to its inherent ease of manipulation and handling, the micromanipulator equipped with a force sensor is a versatile alternative to AFM; (ii) the ability of controlling and monitoring the test within SEM is vital as demonstrated in the case of nanowire twisting, which could otherwise be easily mistaken for the onset of plasticity; and (iii) samples tested in this study were smaller than the ones tested by conventional AFM thus far. Moreover we communicate results obtained on silicon nanowires, which were fabricated by a deep etching technique, described comprehensively in a separate paper [14].

\section{Materials and Methods}

Silicon nanowires were prepared in a monolithic process consisting of the following steps: (1) patterning: a high-resolution lithography providing resist lines on a (100) Si along $\langle 110\rangle$ directions with nominal widths of $20 \mathrm{~nm}$ and $80 \mathrm{~nm}$; (2) definition of a Si precursor line: an anisotropic etching in $\mathrm{Cl}_{2}$ plasma forming a Si protrusion on the surface; (3) sidewall passivation: conformal coating with a low-temperature oxide
(LTO) film and removal of LTO from horizontal surfaces; (4) forming of nanowire: deep reactive ion etching releasing the nanowire by inducing an undercut; and (5) removal of resist and oxide films around the nanowire. An etch depth of $10 \mu \mathrm{m}$ was achieved while nanowires remained intact. The obtained wires are single-crystalline and rigidly attached to silicon anchors at both ends [14]. Figure 1(a) shows two doubleclamped nanowires with triangular supports. It should be emphasized that both the nanowires and microscale supports were fabricated during the same etching process; hence, the technique is monolithic.

Representing the upper and lower boundaries of achievable structures with the aforementioned technology, smallest and largest available nanowires were tested. Exact shape and dimensions of these nanowires were investigated by preparing cross sections with a DualBeam FIB/SEM instrument Quanta 3D FEG from FEI. First the nanowires were protected with a thick platinum coating using the chemical vapor deposition system installed in the FIB. Then the nanowire cross section was prepared like a normal lamella for TEM [15]. Figures 1(b) and 1(c) show the cross-sectional TEM images of both nanowires recorded with a JEOL JEM 2200 FS. The shape resembles a trapezoid with a tip at the bottom. The deviation from the vertical sidewalls is a side effect of the involved reactive ion etching process [16]. The upper width, $w_{\text {up }}$, was measured to be $74 \mathrm{~nm}$ for the largest available nanowire and $35 \mathrm{~nm}$ for the smallest available nanowire. The bottom width, $w_{b}$, amounts to $112 \mathrm{~nm}$ and $66 \mathrm{~nm}$, for the largest and smallest nanowires, respectively. The height, $h$, was $168 \mathrm{~nm}$ for both structures. The lower tip due to the radii of undercut associated with the deep etching is more pronounced in the case of the largest nanowire leading to a maximum height, $h_{\max }$, of $198 \mathrm{~nm}$. Moreover, the TEM images show the crystallinity and the outermost amorphous 


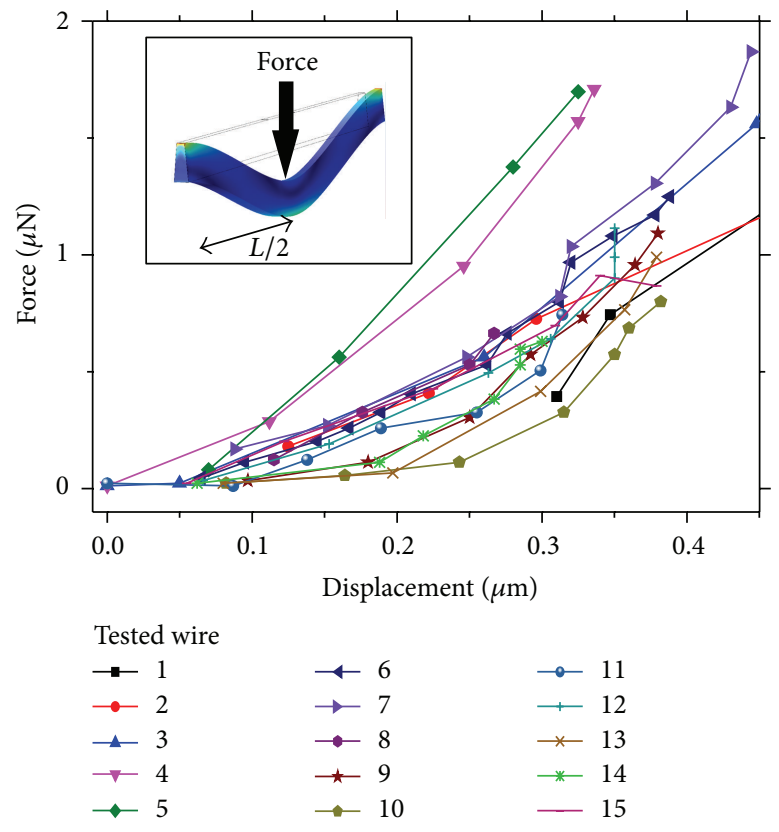

(a)

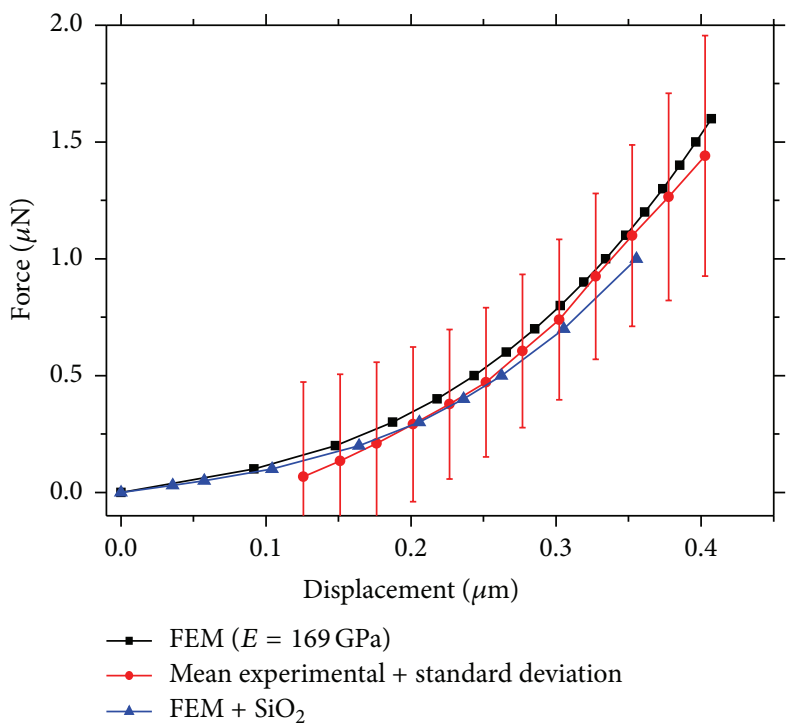

(b)

FIGURE 2: Force-displacement curves for the $74 \mathrm{~nm}$ wide nanowire: (a) results of 15 experiments; the inset shows a schematic of the testing set-up, and (b) overlay of the mean experimental curve and FEM simulations for a modulus of elasticity of $169 \mathrm{GPa}$.

TABLE 1: Measured nanowire geometries.

\begin{tabular}{lcccc}
\hline Nanowire & $w_{\text {up }}[\mathrm{nm}]$ & $w_{b}[\mathrm{~nm}]$ & $h[\mathrm{~nm}]$ & Length $[\mu \mathrm{m}]$ \\
\hline Small & 35 & 66 & 168 & 3 \\
Large & 74 & 112 & 168 & 12 \\
\hline
\end{tabular}

silicon dioxide layer with a uniform thickness of about $4 \mathrm{~nm}$ (Figures 1(c1) and 1(c2)). From the SEM images, a nanowire length of $12 \mu \mathrm{m}$ for the largest nanowire and $3 \mu \mathrm{m}$ for the smallest nanowire was determined. Table 1 provides an overview of the measured values.

A micromanipulator equipped with a cantilever-based force measurement tool from Kleindiek, Germany, was used to characterize the elastic behavior of the double-anchored nanowires. Hence, the nanowires were bent at their midpoint with a silicon tip including a piezoresistive gage (Figure 2(a) inset). Upon contact with the test sample, the piezoresistive gage produces an analog voltage output, which isdue to a former calibration step-converted into a force value recorded with a sampling period of one second. The whole experiment was performed in situ within the vacuum chamber of the FIB/SEM at ambient temperature. From the SEM images it was possible to extract the displacement of the nanowire with an image correlation software (VEDDAC 6) in pixel size precision for each force measurement. Furthermore, one can observe sample behavior throughout the test. In particular the pure elastic loading and unloading without any visible deformation after testing can be observed and the tests provide valuable insight into the reasons of deviations from the expected force-displacement behavior, as described later. Since the nanowires are rigidly anchored to the support by the material itself, insufficient fixing effects can be excluded in the following discussion of the results. In whole 30 nanowires were tested, 15 single wires with $74 \mathrm{~nm}$ width and 16 single wires with $35 \mathrm{~nm}$ width.

\section{Results and Discussion}

Force-displacement curves for the $74 \mathrm{~nm}$ wide and $12 \mu \mathrm{m}$ long nanowires are depicted in Figure 2(a). The curves show an exponential slope as described by Heidelberg et al. [17]. Data scatter can be due to various reasons. First of all, the length measurement error via SEM images can be estimated to be $6 \%$, especially for the displacements around $100 \mathrm{~nm}$ and below. Second, the force measurement error is about $10 \%$ due to uncertainties originating from the former calibration procedure. As silicon is a brittle material, unexpected fracture may occur due to preexisting flaws as observed in some few tests. The deformation of the cantilever itself can be neglected here, because the stiffness of the system was confirmed for much higher loads and changes at the tip could not be observed in the SEM.

To evaluate the experimental results further, finite element model (FEM) simulations were carried out. Nanowires were modeled as double-anchored beams. Nanowire dimensions were extracted from Figures 1(a), 1(b), and 1(c). A linear-elastic material model was utilized with the material constants of single-crystalline silicon along with geometric nonlinearity. A distributed load in the form of uniform pressure was applied over a surface boundary defined on the top surface at the nanowire midspan. The extent of this contact area was determined in accordance with the experimental observations. A commercial finite element software 

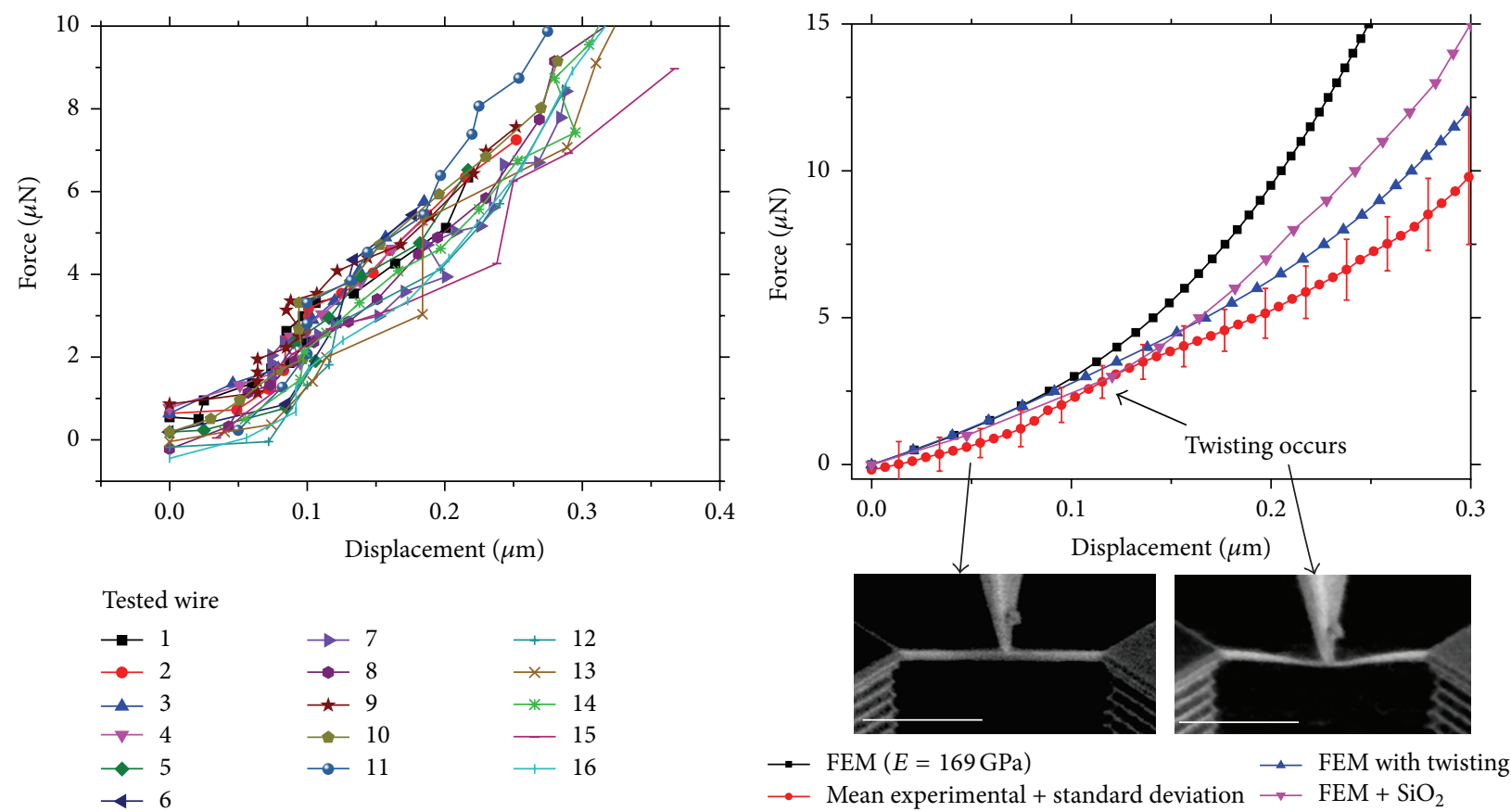

(a)

(b)
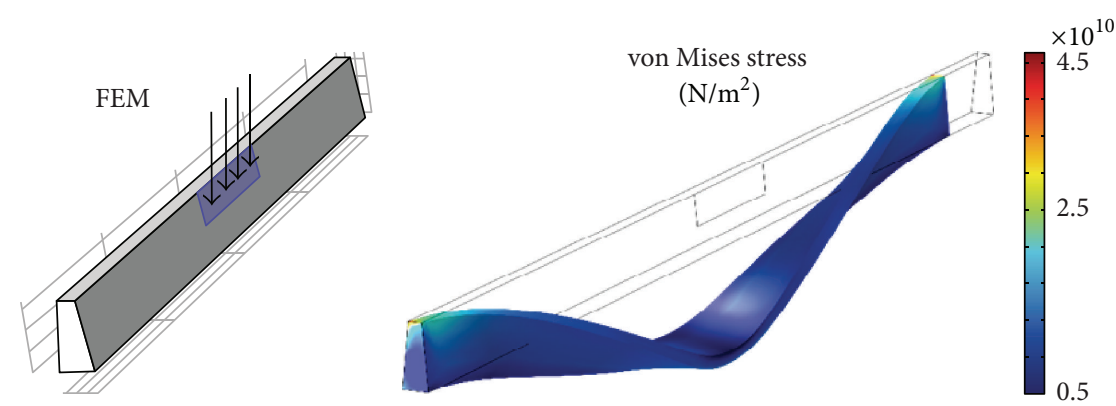

(c)

FIGURE 3: Force-displacement curves for the $35 \mathrm{~nm}$ wide nanowire: (a) results of 16 experiments, (b) overlay of the mean experimental curve and FEM simulations for a modulus of elasticity of $169 \mathrm{GPa}$, where nanowires exhibit twisting at a deflection value beyond $100 \mathrm{~nm}$ (see SEM images below; scale bar: $2 \mu \mathrm{m}$ ) leading to a different slope in the loading curve, and (c) FEM model of the twisting phenomenon along with the deformed nanowire configuration.

was utilized for modeling of $19 \times 10^{3}$ tetrahedral and $6 \times 10^{3}$ triangular elements with about $95 \times 10^{3}$ degrees of freedom. The maximum mesh density was imposed at the contact surface. As experiments involved large displacements, the model was validated first with a closed-form solution available in the literature [17]. Following model validation, a parametric study was carried out within the experimental force range. For each applied force value nanowire displacement at the midspan was recorded.

The simulated force-displacement curve for the largest nanowire $\left(w_{\text {up }}=74 \mathrm{~nm}\right)$ is given in Figure 2(b) with solid rectangles, where the modulus of elasticity was taken as $169 \mathrm{GPa}$. The mean experimental curve with error bars is provided in Figure 2(b) as well. Considering the uncertainty budget of the method as described above, a very close correspondence is evident between experimental results and simulations.
Further tests were performed on the smallest available nanowires $\left(w_{\mathrm{up}}=35 \mathrm{~nm}\right)$ (Figure $\left.3(\mathrm{a})\right)$. A comparison of the mean experimental curve with simulation results is provided in Figure 3(b). As opposed to the previous case, a significant deviation between experiments and simulations is evident beyond a displacement of $100 \mathrm{~nm}$. A plastic deformation of the wire can be excluded here, because the displacement analysis yields a full recovery of the wire after loading. Instead a closer look through in situ observations in SEM reveals a twisting phenomenon (Figure 3(b) below). As it became more difficult to establish contact with the nanowire at the narrow space on its top surface with a relatively larger silicon tip of the force sensor, the nanowire is thought to have been loaded along its side surface creating a net torsion. The ability of online monitoring is critical in such circumstances, as in the absence of direct observation twisting could be mistaken for other phenomena such as plasticity. 
To confirm the effect of twisting on the nanowire bending behavior, FEM simulations were again carried out. Modeling was similar to that of the previous nanowire geometry. This time $4 \times 10^{3}$ tetrahedral and $2 \times 10^{3}$ triangular elements were utilized with the number of degrees of freedom amounting to $22 \times 10^{3}$. Load was applied not on the top surface of the nanowire but across a contact area defined along the top periphery of the sidewall, thereby creating a torque. Both the contact area with the distributed load and the deformed configuration of a twisted nanowire are depicted in Figure 3(c). Simulation results for the force-displacement behavior in the presence of twisting are included in Figure 3(b) along with the case of ideal loading. Results confirm that twisting is accompanied by a considerable reduction in bending stiffness. Such twisting in Si nanowires was recently reported to be accompanied by a significant change in nanowire transport properties [18]. Hence, the proposed testing platform can be used in the future to measure nanowire transport properties under loading. Furthermore, it is to be noted that twisting was not observed with the larger nanowires due to the relative ease of positioning of the force sensor tip on the nanowire sample. Although twisting was observed at displacements of the order of $100 \mathrm{~nm}$ in the case of smaller nanowires, such displacements could not be reliably resolved in SEM. Hence, larger displacements were used to study the elastic behavior of the $35 \mathrm{~nm}$ wide nanowires more accurately. These large displacements were still pure elastic since the wire returns to the original position after testing.

Figures 2(b) and 3(b) further emphasize the effect of native oxide on the force-displacement curve. If the existence of this $4 \mathrm{~nm}$ thick amorphous silicon dioxide layer is taken into account, a reduction of stiffness is obtained. This is achieved by modeling the nanowire as a bilayer structure with a uniform silicon dioxide layer around the Si core in accordance with the TEM observations given in Figure 1. A modulus of elasticity of $70 \mathrm{GPa}$ was taken for the oxide layer. It is expected that the reduction of bending rigidity is more pronounced in the case of the smaller nanowire, as the volume ratio of silicon dioxide to silicon is increased. Indeed the loss of rigidity can be observed in FEM simulations in both cases, but the effect is more obvious for the smaller nanowire. This effect further explains the slightly lower rigidity of the small nanowire compared to that of the larger nanowire.

A comparison of the results with data from the literature reveals good agreement. Sundararajan and Bhushan measured $182 \mathrm{GPa}$ for $\mathrm{E}\langle 110\rangle$ [10]. Herein the authors present linear force-displacement curves for wires twice as large as ours compared to exponential curves for the smaller wires as shown in this study and in the work of Heidelberg et al. [17]. A match was also found with the study of Paulo et al. (E $\langle 111\rangle, 186 \mathrm{GPa})$ [8], Gordon et al. (100-180 GPa) [9], and $\mathrm{Zhu}$ et al. (166 GPa) [11]. A slightly lower modulus of elasticity of $147 \mathrm{GPa}$ had to be assumed by Wang et al. for modeling a compression test with a flat intender performed on the cylindrical face of a silicon nanowire [13]. Taking into account that all these tests were applied to silicon nanowires prepared by different processes, the match is satisfying. All these studies exclude a size effect within the measured size range. In contrast Sadeghian et al. [19] found a clearly reduced modulus of elasticity for silicon $\langle 110\rangle$ nanobeams thinner than $150 \mathrm{~nm}$. Similar results are shown by Li et al. for ultrathin silicon cantilevers [20]. Moreover a size effect was observed by Zhu et al. [11], but only for wire diameters smaller than $25 \mathrm{~nm}$. Our findings fit the latter results indicating no size effect for structures bigger than $25 \mathrm{~nm}$.

\section{Conclusions}

It has been demonstrated that it is possible to prepare double-anchored silicon nanowires, where nanowires and their supports were etched from the same silicon crystal. This eliminated any interface compliance, which would hinder the accuracy of nanomechanical characterization results. Nanowire cross sections had trapezoidal or pentagonal shapes. The maximum nanowire width utilized in this study was $74 \mathrm{~nm}$, whereas a minimum of $35 \mathrm{~nm}$ could be achieved. Three-point bending tests were conducted on the nanowires within the FIB/SEM chamber via a force sensor probe. The measured force-displacement curves yielded good correlation in comparison to simulated force-displacement curves with the modulus of elasticity taken as the bulk $\langle 110\rangle$ value of $169 \mathrm{GPa}$ [21]. Thus this simple force measurement tool represents a cost-saving alternative to common techniques. The method is applicable to even smaller objects, if available. Good compliance with already published AFM data proves the reliability of the method. Small deviations between successive tests can be explained by uncertainties of tip positioning and, specifically in the case of smaller nanowires, twisting of the nanowire. A further slight systematic deviation observed for the smallest nanowire was attributed to the influence of the very small native oxide layer. The important impact of geometry was investigated comprehensively, both experimentally (TEM of cross sections) and numerically. No size effect on the elastic behavior of silicon was found within the considered size range.

\section{Competing Interests}

The authors declare that they have no competing interests.

\section{Acknowledgments}

This research is supported by the European Union by funding the European Metrology Research Programme (EMRP) project "Traceable Measurement of Mechanical Properties of Nano-Objects (MechProNO)." The EMRP is jointly funded by the EMRP participating countries within EURAMET and the European Union. Moreover this work is supported by Tubitak under Grant no. 112E058 and Koç University-Istanbul Rotary Club Fundamental Research Seed Fund Program. The authors acknowledge the help from CMI staff, Lausanne, Switzerland. M. Nasr Esfahani and G. Nadar are thanked for their help in simulations. 


\section{References}

[1] Y.-S. Kim, S. H. Yang, K. W. Yang, and N. G. Dagalakis, “Design of MEMS vision tracking system based on a micro fiducial marker," Sensors and Actuators A: Physical, vol. 234, pp. 48-56, 2015.

[2] X. Zhang, C. Duan, L. Liu, X. Li, and H. Xie, "A non-resonant fiber scanner based on an electrothermally-actuated MEMS stage," Sensors and Actuators A: Physical, vol. 233, pp. 239-245, 2015.

[3] P. Helistö, H. Sipola, H. Seppä, and A. J. Manninen, "MEMSbased voltage detector," Sensors and Actuators, A: Physical, vol. 234, pp. 99-103, 2015.

[4] B. E. Alaca, "Integration of one-dimensional nanostructures with microsystems: an overview," International Materials Reviews, vol. 54, no. 5, pp. 245-282, 2009.

[5] P. E. Allain, A. Bosseboeuf, F. Parrain, S. Maaroufi, P. Coste, and A. Walther, "Large range MEMS motion detection using integrated piezo-resistive silicon nanowire," in Proceedings of the IEEE 25th International Conference on Micro Electro Mechanical Systems (MEMS '12), pp. 1320-1323, Paris, France, JanuaryFebruary 2012.

[6] N. Geyer, N. Wollschläger, B. Fuhrmann et al., "Influence of the doping level on the porosity of silicon nanowires prepared by metal-assisted chemical etching," Nanotechnology, vol. 26, no. 24, Article ID 245301, 2015.

[7] S. Hoffmann, I. Utke, B. Moser et al., "Measurement of the bending strength of vapor-liquid-solid grown silicon nanowires," Nano Letters, vol. 6, no. 4, pp. 622-625, 2006.

[8] A. S. Paulo, J. Bokor, R. T. Howe et al., "Mechanical elasticity of single and double clamped silicon nanobeams fabricated by the vapor-liquid-solid method," Applied Physics Letters, vol. 87, no. 5, Article ID 053111, 2005.

[9] M. J. Gordon, T. Baron, F. Dhalluin, P. Gentile, and P. Ferret, "Size effects in mechanical deformation and fracture of cantilevered silicon nanowires," Nano Letters, vol. 9, no. 2, pp. 525529, 2009.

[10] S. Sundararajan and B. Bhushan, "Development of AFM-based techniques to measure mechanical properties of nanoscale structures," Sensors and Actuators, A: Physical, vol. 101, no. 3, pp. 338-351, 2002.

[11] Y. Zhu, F. Xu, G. Qin, W. Y. Fung, and W. Lu, "Mechanical properties of vapor-Liquid-Solid synthesized silicon nanowires," Nano Letters, vol. 9, no. 11, pp. 3934-3939, 2009.

[12] T. Fujii, K. Sudoh, S. Sakakihara, M. Naito, S. Inoue, and T. Namazu, "Nano-scale tensile testing and sample preparation techniques for silicon nanowires," Japanese Journal of Applied Physics, vol. 52, no. 11, Article ID 110118, 2013.

[13] Z. Wang, W. M. Mook, C. Niederberger, R. Ghisleni, L. Philippe, and J. Michler, "Compression of nanowires using a flat indenter: diametrical elasticity measurement," Nano Letters, vol. 12, no. 5, pp. 2289-2293, 2012.

[14] Z. Tasdemir, N. Wollschläger, W. Österle, Y. Leblebici, and B. E. Alaca, "A deep etching mechanism for trench-bridging silicon nanowires," Nanotechnology, vol. 27, no. 9, Article ID 095303, 2016.

[15] M. Schaffer, B. Schaffer, and Q. Ramasse, "Sample preparation for atomic-resolution STEM at low voltages by FIB," Ultramicroscopy, vol. 114, pp. 62-71, 2012.

[16] H. V. Jansen, M. J. de Boer, S. Unnikrishnan, M. C. Louwerse, and M. C. Elwenspoek, "Black silicon method: X. A review on high speed and selective plasma etching of silicon with profile control: an in-depth comparison between Bosch and cryostat DRIE processes as a roadmap to next generation equipment," Journal of Micromechanics and Microengineering, vol. 19, Article ID 033001, 2009.

[17] A. Heidelberg, L. T. Ngo, B. Wu et al., "A generalized description of the elastic properties of nanowires," Nano Letters, vol. 6, no. 6, pp. 1101-1106, 2006.

[18] J. C. Garcia and J. F. Justo, “Twisted ultrathin silicon nanowires: a possible torsion electromechanical nanodevice," Europhysics Letters, vol. 108, no. 3, Article ID 36006, 2014.

[19] H. Sadeghian, C. K. Yang, J. F. L. Goosen et al., "Characterizing size-dependent effective elastic modulus of silicon nanocantilevers using electrostatic pull-in instability," Applied Physics Letters, vol. 94, no. 22, Article ID 221903, 2009.

[20] X. Li, T. Ono, Y. Wang, and M. Esashi, "Ultrathin singlecrystalline-silicon cantilever resonators: fabrication technology and significant specimen size effect on Young's modulus," Applied Physics Letters, vol. 83, no. 15, pp. 3081-3083, 2003.

[21] M. A. Hopcroft, W. D. Nix, and T. W. Kenny, "What is the Young's modulus of silicon?" Journal of Microelectromechanical Systems, vol. 19, no. 2, pp. 229-238, 2010. 

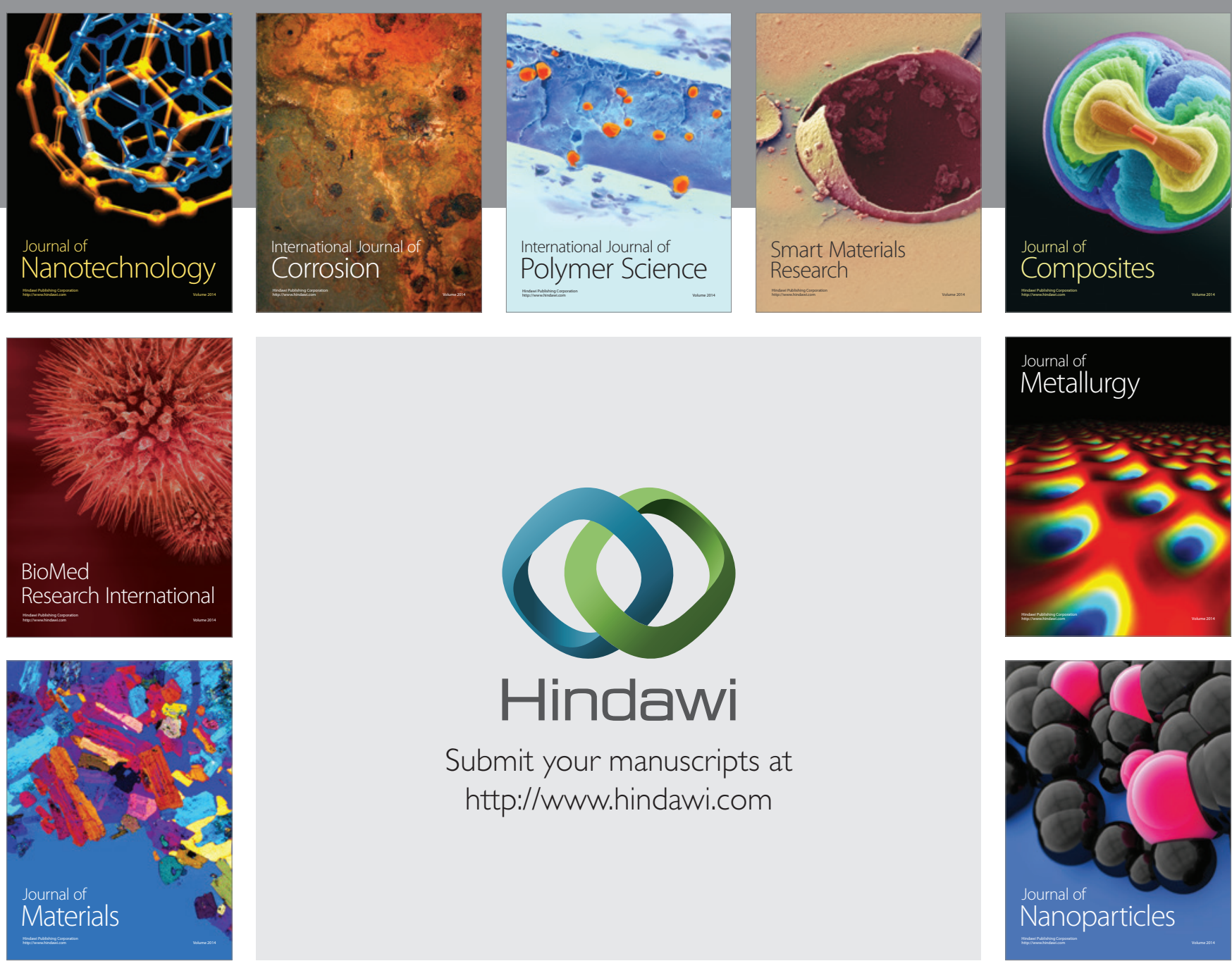

\section{Hindawi}

Submit your manuscripts at

http://www.hindawi.com

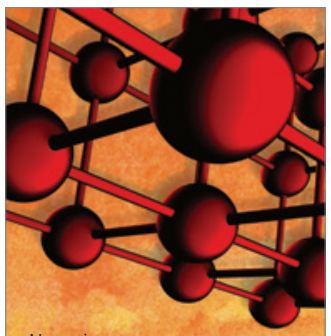

Materials Science and Engineering
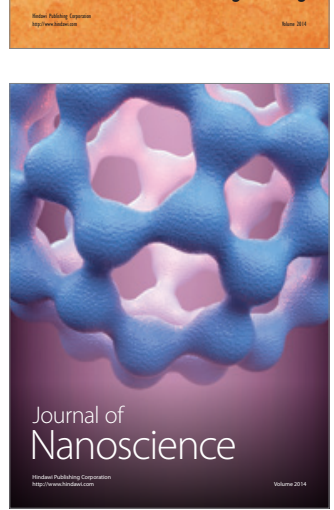
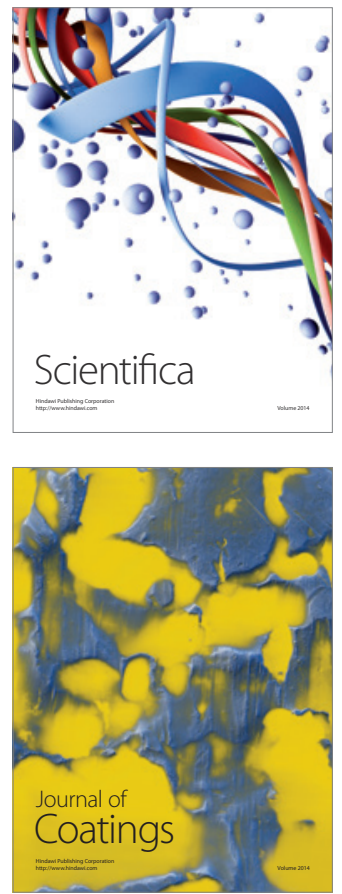
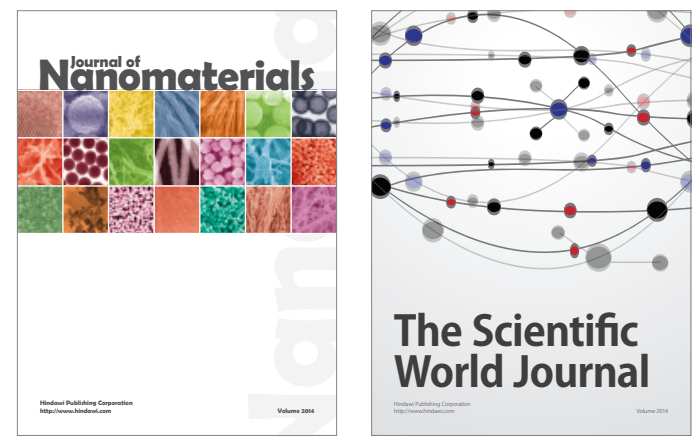

The Scientific World Journal
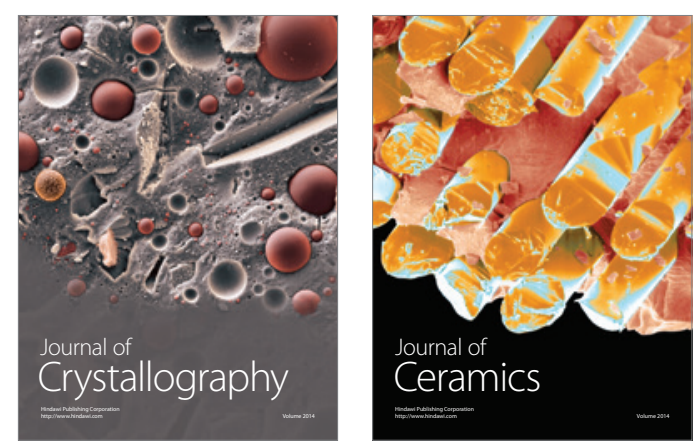
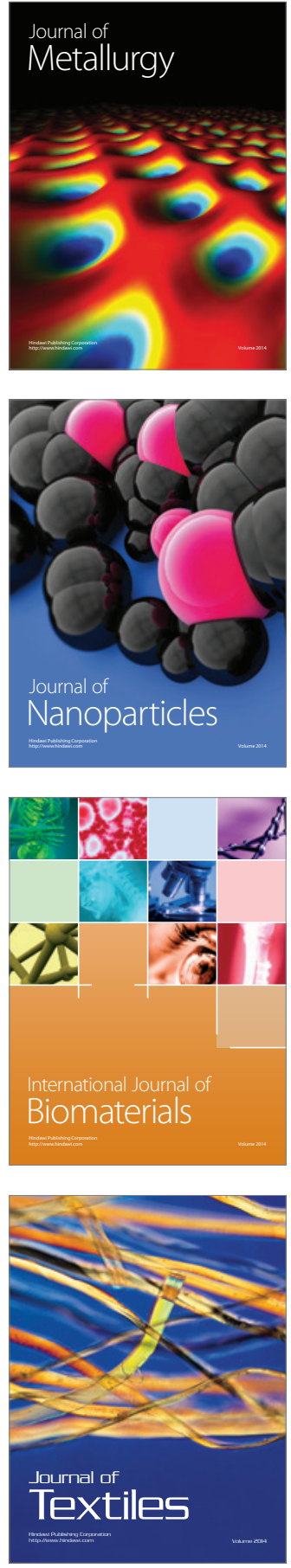